\title{
Implicações audiológicas do diabetes mellitus e da hipertensão arterial: uma revisão sistemática
}

\author{
Audiological implications of diabetes \\ mellitus and arterial hypertension: \\ a systematic review
}

\section{Implicaciones audiológicas de la diabetes mellitus y la hipertensión arterial: una revisión sistemática}

\author{
Graziela Mackowiesky Brigido Bernardo* \\ Suelen Bernardo Guckert* \\ Karina Mary Paiva* \\ Fernanda Soares Aurélio Patatt* \\ Patricia Haas*
}

\section{Resumo}

Introdução: Dentre as doenças crônicas não transmissíveis (DCNT) mais prevalentes, destacamse o Diabetes Mellitus (DM) e a Hipertensão Arterial Sistêmica (HAS). Estas DCNT se relacionam ao estilo de vida, idade e gênero, e podem estar associadas a alterações audiológicas. Objetivo: Evidenciar os estudos já existentes na literatura a respeito da DM e a HAS e a sua relação com a perda auditiva. Estratégia de pesquisa: As buscas por artigos científicos ocorreram nas bases de dados eletrônicos Medline (Pubmed), LILACS e SciELO. Critério de seleção: Foram incluídos estudos sobre a relação da perda auditiva com a DM e HAS publicados nos últimos cinco anos, e, excluídas revisões de literatura, revisões sistemáticas e meta-análises. Resultados: Foram selecionados nove estudos evidenciando que

* Universidade Federal de Santa Catarina - UFSC - Santa Catarina - Brasil

\section{Contribuição dos autores:}

GMBB: Contribuições substanciais à concepção ou ao projeto do trabalho, à aquisição, à análise, a interpretação dos dados e a redação. SBG: Contribuições substanciais à concepção ou ao projeto do trabalho, à aquisição, à análise, a interpretação dos dados e a redação. KMP: Contribuições substanciais à concepção ou ao projeto do trabalho, à aquisição, à análise, a interpretação dos dados, à redação e a aprovação final.

FSAP: Contribuições substanciais à concepção ou ao projeto do trabalho, à aquisição, à análise, a interpretação dos dados, à redação e a aprovação final.

PH: Contribuições substanciais à concepção ou ao projeto do trabalho, à aquisição, à análise, a interpretação dos dados, à redação e a aprovação final.

E-mail para correspondência: Sabrina Vilanova Cardoso - sabrinavilanova@hotmail.com Recebido: 22/04/2019

Aprovado: $12 / 04 / 2020$ 
a DM e/ou a HAS podem ser possíveis fatores de risco para o desenvolvimento da perda auditiva; para isso foram realizadas avaliações e comparações de grupos de pessoas com e sem tais fatores de risco. Outras comorbidades podem ser determinantes para a perda auditiva. Após a análise, verificou-se que existe uma relação entre a HAS, DM e a perda auditiva, principalmente em altas frequências. Não foi observada nenhuma diferença entre as orelhas. Conclusão: Existe relação entre DM e HAS com a perda auditiva, tanto em adultos quanto em idosos. O comprometimento auditivo mostrou-se maior nos sujeitos que apresentavam DM e HAS associados, sugerindo um efeito sinérgico das duas doenças crônicas sobre a audição. A alteração auditiva do tipo neurossensorial prevaleceu.

Palavras-chave: Diabetes Mellitus; Hipertensão; Perda Auditiva.

\section{Abstract}

Introduction: Among the most prevalent chronic non-communicable diseases (NCDs), Diabetes Mellitus (DM) and Systemic Arterial Hypertension (SAH) stand out. These NCDs are related to lifestyle, age and gender, and may be associated with audiological changes. Objective: To highlight the studies that already exist in the literature regarding DM and SAH and their relationship with hearing loss. Research strategy: Searches for scientific articles occurred in the electronic databases Medline (Pubmed), LILACS and SciELO. Selection criteria: Studies on the relationship between hearing loss and DM and SAH published in the last five years were included, and literature reviews, systematic reviews and meta-analysis were excluded. Results: Nine studies were selected showing that DM and / or SAH may be possible risk factors for the development of hearing loss. For this purpose, evaluations and comparisons of groups of people with and without such risk factors were carried out. Other comorbidities can be determinant for hearing loss. After the analysis, it was found that there is a relationship between SAH, DM and hearing loss, especially at high frequencies. No difference was observed between the ears. Conclusion: There is a relationship between DM and SAH with hearing loss, both in adults and in the elderly. Auditory impairment was shown to be greater in subjects with associated DM and $\mathrm{SAH}$, suggesting a synergistic effect of the two chronic diseases on hearing. The hearing loss of the sensorineural type prevailed.

Keywords: Diabetes Mellitus; Hypertension; Hearing Loss.

\section{Resumen}

Introducción: Entre las enfermedades crónicas no transmisibles (ENT) más prevalentes, destacan la diabetes mellitus (DM) y la hipertensión arterial sistémica (HSA). Estas ENT están relacionadas con el estilo de vida, la edad y el género, y pueden estar asociadas con cambios audiológicos. Objetivo: destacar los estudios que ya existen en la literatura sobre DM y SAH y su relación con la pérdida auditiva. Estrategia de investigación: Se realizaron búsquedas de artículos científicos en las bases de datos electrónicas Medline (Pubmed), LILACS y SciELO. Criterios de selección: Se incluyeron los estudios sobre la relación entre la pérdida auditiva y la DM y la HSA publicados en los últimos cinco años, y se excluyeron las revisiones de literatura, revisiones sistemáticas y metanálisis. Resultados: Se seleccionaron nueve estudios que demuestran que la DM y / o la HSA pueden ser posibles factores de riesgo para el desarrollo de la pérdida auditiva, para lo cual se llevaron a cabo evaluaciones y comparaciones de grupos de personas con y sin dichos factores de riesgo. Otras comorbilidades pueden ser determinantes para la pérdida auditiva. Después del análisis, se encontró que existe una relación entre SAH, DM y pérdida de audición, especialmente a altas frecuencias. No se observó diferencia entre las orejas. Conclusión: Existe una relación entre DM y SAH con pérdida auditiva, tanto en adultos como en ancianos. Se demostró que la deficiencia auditiva era mayor en sujetos con DM y SAH asociadas, lo que sugiere un efecto sinérgico de las dos enfermedades crónicas en la audición. La pérdida auditiva del tipo neurosensorial prevaleció.

Palabras clave: Diabetes Mellitus; Hipertensión; Pérdida auditiva. 


\section{Introdução}

Atualmente cerca de um terço das mortes globais ocorrem a partir do acometimento de doenças crônicas não transmissíveis (DCNTs), que estão associadas a condições de vida desfavoráveis, tais como baixa escolaridade, renda familiar per capita baixa e estilo de vida não saudável como o tabagismo, o alcoolismo, o sedentarismo, e o estresse ${ }^{1,2}$.

As DCNTs caracterizam-se por apresentar etiologia múltipla, períodos de latência longos, origem não infecciosa e também por associarem-se a deficiências e incapacidades funcionais. Entre as DCNTs mais conhecidas e prevalentes estão o Diabetes Mellitus (DM) e a Hipertensão Arterial Sistêmica (HAS) 3 .

A DM é conhecida como uma doença metabólica caracterizada por hiperglicemia e associada a complicações, disfunções e insuficiência de vários órgãos, dentre eles: olhos, rins, nervos, cérebro, coração e vasos sanguíneos. Podem ocorrer defeitos de secreção e/ou ação da insulina e até destruição das células produtoras desse hormônio ${ }^{4}$.

Acredita-se que uma das implicações audiológicas em indivíduos com diabetes seja a angiopatia, que pode interferir no suprimento de nutrientes e oxigênio da cóclea. Além das alterações cocleares, o DM também pode causar degeneração secundária do oitavo nervo craniano (VII), provocando perdas auditivas neurais ${ }^{1,5}$.

Com relação à HAS há outros fatores de risco que são concomitantes que aumentam a probabilidade do indivíduo vir a ter uma doença cardiovascular $^{1,6}$, como idade, gênero, histórico familiar, tabagismo, alcoolismo e sedentarismo. De acordo com a Organização Mundial da Saúde (OMS) $(2015)^{7}$, o aumento da prevalência de HAS pode estar associada ao envelhecimento, podendo este ser o principal fator determinante desse acréscimo uma vez que muitos desses fatores são modificáveis.

A hipertensão é um dos distúrbios vasculares mais comuns, que pode facilitar alterações estruturais do coração e vasos sanguíneos. A pressão elevada no sistema vascular pode resultar em hemorragias na orelha interna, que, assim como outras partes do corpo, recebe suprimento de sangue derivado da artéria cerebelar inferior anterior, que se divide e dá suporte a outros ramos da orelha, podendo culminar em perdas auditivas súbitas ou progressivas $^{1,8}$.
Esta pesquisa tem por objetivo evidenciar estudos sobre Diabetes Mellitus e Hipertensão Arterial Sistêmica e sua relação com a perda auditiva, em uma perspectiva atual.

\section{Estratégia de pesquisa}

Trata-se de revisão sistemática, conduzida conforme as recomendações do Preferred Reporting Items for Systematic Reviews and Meta-Analyses (PRISMA) ${ }^{9}$.

A busca pelos artigos foi realizada nas seguintes bases de dados: MEDLINE (via PubMed) (https://www.ncbi.nlm.nih. gov/pubmed/), Scientific Electronic Library Online (SciELO) (http:// www. scielo.br/) e Literatura Latino-Americana e do Caribe em Ciências da Saúde (LILACS) (http:// lilacs.bvsalud.org/). A estratégia de busca para o MEDLINE foi a seguinte: ("diabetes mellitus" [Mesh]) AND ("hypertension" [Mesh]) AND ("hearing loss" [Mesh]). Para as bases Scopus, SciELO e LILACS utilizou-se a combinação de palavras/termos: ("diabetes mellitus" [DeCS] E "hipertensão" [DeCS] E "perda auditiva" [DeCS]). O período de busca foi com os artigos publicados entre 2014 e 2019. Os artigos incluídos nesta revisão sistemática foram selecionados por dois autores independentes, e foram divididos quatro passos para a recuperação dos artigos.

No primeiro passo, os artigos foram identificados por meio da busca eletrônica, organizados e revisados para verificação de duplicidade pelos dois autores de forma independente. No segundo passo, foram analisados de forma independente os títulos dos artigos. Foram excluídos os artigos que não atendiam algum critério de inclusão. No terceiro passo, os resumos dos artigos selecionados no segundo passo foram examinados. Os artigos que não continham características da população estudada foram excluídos. Havendo insuficiência de dados, o resumo era deixado para a próxima etapa de avaliação. No quarto passo, os textos completos dos artigos foram recuperados e revisados. Foram selecionados os que apresentavam dados a respeito da relação da DM e a HAS com a perda auditiva.

A pesquisa foi estruturada e organizada na forma PICOS (Quadro 1), que representa um acrônimo para População alvo, a Intervenção, Comparação e "Outcomes" (desfechos), Study. 
Quadro 1. Descrição da estratégia PICOS.

\begin{tabular}{|c|c|c|}
\hline Acrônimo & Definição & Descrição \\
\hline P & Paciente ou problema & Relação da diabetes mellitus e hipertensão arterial sistêmica \\
com a perda auditiva
\end{tabular}

\section{Critérios de seleção}

Os artigos selecionados para essa revisão foram os que atenderam aos seguintes critérios de inclusão: indivíduos com perda auditiva em decorrência de doenças crônicas não transmissíveis como a diabetes e hipertensão; idiomas português, inglês e espanhol sem restrição de localização, porém com restrição de período de publicação, sendo incluídos estudos publicados de 2014 a 2019.
Foram excluídos estudos com as seguintes características: revisões de literatura, revisões sistemáticas, meta-análises, intervenções ou processo de randomização, estudos observacionais, cartas ao editor, diretrizes e resumos. Estudos mal descritos ou que foram pouco claros ou indisponíveis também não foram aceitos nesta pesquisa. Abaixo, pode-se verificar a síntese dos critérios de inclusão e exclusão da presente revisão (Quadro 2).

Quadro 2. Critérios de inclusão e exclusão.

\begin{tabular}{|c|c|}
\hline \multicolumn{2}{|c|}{ Critérios de Inclusão } \\
\hline Delineamento & Estudos de caso, estudos longitudinais, estudos transversais e estudos de prevalência \\
\hline Localização & Sem restrição \\
\hline Idioma & Sem restrição \\
\hline Ano & De 2014 até 2019 \\
\hline \multicolumn{2}{|c|}{ Critérios de Exclusão } \\
\hline Delineamento & Revisões de literatura, revisões sistemáticas e meta-análises \\
\hline Estudos & Estudos pouco claros ou mal descritos ou inadequados \\
\hline Forma de publicação & Somente em resumos \\
\hline
\end{tabular}

\section{Análise de dados}

Dois pesquisadores independentes analisaram os resultados de pesquisa para encontrar estudos potencialmente elegíveis. Inicialmente foram encontrados 137 artigos, posteriormente, realizou-se a exclusão por repetição, restando 127 artigos; em seguida, os títulos foram analisados e excluídos quando não se encaixavam com os critérios, res- tando 30 artigos; sucessivamente os resumos foram analisados e apenas os que fossem potencialmente elegíveis foram selecionados, sendo estes 18 artigos. Com base nos resumos, os artigos foram selecionados para leitura integral, restando somente nove artigos que atendiam a todos os critérios pré-determinados (Figura 1). 


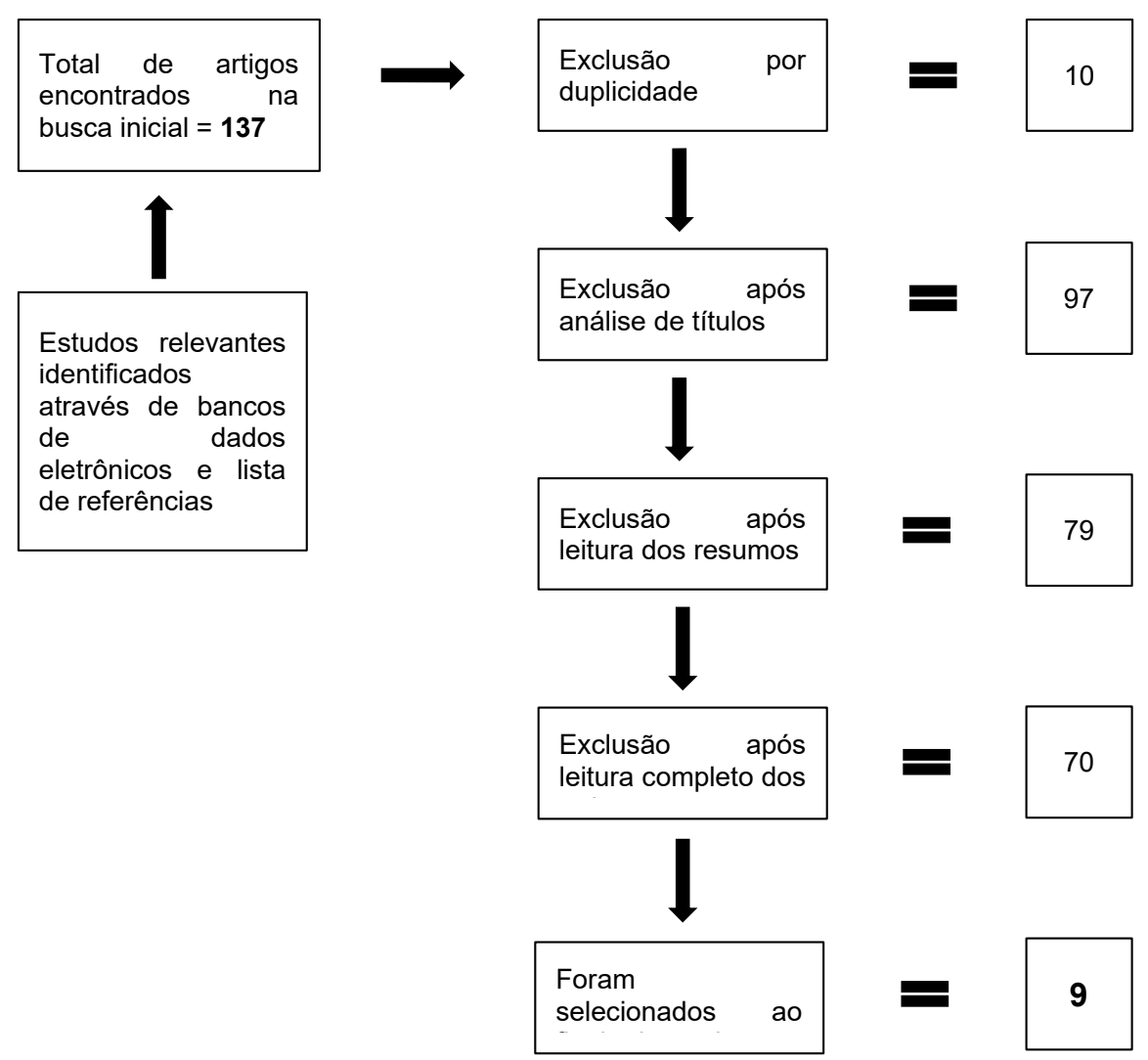

Figura 1. Busca e análise dos artigos.

\section{Resultados}

A partir das buscas realizadas, foram selecionados nove artigos que contemplavam todos os critérios de inclusão (Tabela 1). Os estudos selecionados evidenciaram a DM e a HAS como possíveis fatores de risco para o desenvolvimento da perda auditiva ${ }^{10-12}$. Pacientes com DM possuem um risco maior de desenvolver uma perda auditi$\mathrm{va}^{12}$ do tipo sensorioneural ${ }^{11,12,14,16}$. Alguns estudos destacaram que o tabagismo ${ }^{12}$, o elevado Índice de Massa Corpórea (IMC) ${ }^{10}$, a exposição ao ruído e a idade elevada ${ }^{14}$ também podem ser determinantes para a perda auditiva (Tabela1).

No que diz respeito à metodologia adotada pelos pesquisadores, destacam-se os estudos transversais ${ }^{10,13,14,15,16,18}$, sendo encontradas também pesquisas de coorte multicêntrica ${ }^{17}$, caso-controle ${ }^{12}$ e retrospectiva ${ }^{11}$. Quanto ao público estudado, em seis pesquisas os autores optaram por avaliar adultos e/ou idosos de 20 a 87 anos de idade ${ }^{10,12,13,15,16,18}$
, enquanto outros três estudos realizaram sua pesquisa apenas com a população idosa (idade $\geq a$ 60 anos) ${ }^{11,14,17}$. O número de sujeitos selecionados para as pesquisas variou de 80 a $37.773^{10-12,14-18}$, sendo que uma delas não mencionou o número de indivíduos inclusos ${ }^{13}$. Em relação ao sexo dos participantes, destaca-se o feminino ${ }^{10,13,14,16}$ porém, não foram todos os autores que mencionaram o sexo dos indivíduos estudados ${ }^{11,17,18}$. Um estudo demonstrou distribuição muito semelhante entre os $\operatorname{sexos}^{12}$ e um evidenciou número mais expressivo de participantes do sexo masculino ${ }^{15}$.

A fim de avaliar a saúde física e auditiva dos participantes de cada um dos estudos selecionados para esta revisão de literatura, foi realizada uma série de avaliações. Observou-se uma diferença entre os procedimentos utilizados pelos diferentes autores, dentre eles pode-se apontar a criação e a aplicação de questionário sociodemográfico ${ }^{13}$, testes para aferir a pressão arterial ${ }^{15}$, exame de sangue $^{15,18}$, testes físicos ${ }^{13}$, glicose ${ }^{18}$, avaliação do 
Índice de Massa Corpórea (IMC) $)^{10,13,16}$, ressonância magnética $^{12}$, exame otoscópico ${ }^{10,14,16}$, meatoscopia $^{17}$, audiometria tonal limiar ${ }^{10-18}$, logoaudiometria $^{11,12,17,18}$, imitanciometria ${ }^{11,12,17,18}$ e potencial evocado auditivo de tronco encefálico ${ }^{12}$.

Grande parte dos estudos selecionados para esta revisão realizou o acompanhamento de dois ou mais grupos para mensurar se existe relação desses fatores de risco com a perda auditiva. Alguns estudos foram compostos por, pelo menos, um grupo com DM ou HAS, e/ou um grupo com sujeitos que tem os dois fatores associados (DM+HAS), bem como por um grupo sem estas variáveis, denominado, pelos autores, como grupo controle $\mathrm{e}^{11,16-18}$. Outro estudo, no qual o foco principal foi evidenciar a perda auditiva relacionada a DM, separou um grupo com perda auditiva e outro sem perda auditiva ${ }^{12}$.

Outros aspectos analisados foram o diagnóstico prévio das patologias ${ }^{13,15-18}$ e o tempo em que os participantes haviam recebido esse diagnóstico ${ }^{17,18}$. Cinco estudos descreveram os procedimentos e parâmetros para a realização do diagnóstico ${ }^{13,15-18}$ e um destes, cuidadosamente, dividiu subgrupos separando esses indivíduos de acordo com a idade em que recebeu o diagnóstico ${ }^{17}$. $\mathrm{O}$ tempo de diagnóstico dessas patologias variou, sendo para DM seis anos, para HAS 16 anos e DM+HAS 14 anos $^{17}$.
Após acompanhamento audiológico, alguns estudos mostraram relação significante entre a HAS e/ou DM e a perda auditiva $a^{10,14,15,17}$, principalmente em altas frequências ${ }^{14,15,17}$; entretanto, o comprometimento das frequências médias e baixas também foi constatado ${ }^{11,17}$. Um estudo constatou limiares auditivos e resultados dos testes de fala significativamente piores no grupo com DM do que no grupo sem DM; no entanto, esta diferença não se manteve após o ajuste para idade, sexo e presença de hipertensão, bem como não foi verificada associação entre a duração do diabetes e os limiares auditivos após o referido ajuste ${ }^{18}$. Um maior comprometimento auditivo nos grupos cujos sujeitos apresentavam DM e HAS associados foi evidenciado em algumas publicações, sugerindo um efeito sinérgico das duas doenças crônicas sobre a audição $0^{11,13,16,17}$. A perda auditiva em pacientes com DM tipo 2 foi agravada pela hipertensão e pelo baixo controle glicêmico ${ }^{16}$.

Quanto ao tipo e grau de perda auditiva, destaca-se a perda auditiva do tipo sensorioneural ${ }^{11,14,16}$, bilateral ${ }^{14}$, de grau leve ${ }^{14}$ ou moderado ${ }^{16}$, aumentando progressivamente de acordo com a idade ${ }^{13,14,15} \mathrm{e}$ na presença de outros fatores ${ }^{9,15}$. Não se observou diferença estatística em relação à idade, gênero dos participantes ${ }^{10-18} \mathrm{e} / \mathrm{ou}$ em relação a orelha direita e esquerda $^{11,14}$.

Tabela 1. Síntese dos artigos incluídos na revisão.

\begin{tabular}{|c|c|c|c|c|}
\hline Autor/Ano & Título & Objetivo & Metodologia & Resultados \\
\hline $\begin{array}{c}\text { Stephen } \\
\text { Semen } \\
\text { Yikawe; } \\
\text { Kufre Robert } \\
\text { Iseh; } \\
\text { Anas Ahmad } \\
\text { Sabir; } \\
\text { Mfon Ime } \\
\text { Inoh; } \\
\text { Joseph } \\
\text { Hassan } \\
\text { Solomon; } \\
\text { Nasiru Aliyu / } \\
2018\end{array}$ & $\begin{array}{l}\text { Cardiovascular } \\
\text { risk factors } \\
\text { and hearing } \\
\text { loss among } \\
\text { adults in } \\
\text { a tertiary } \\
\text { center of } \\
\text { Northwestern } \\
\text { Nigeria }^{10}\end{array}$ & $\begin{array}{c}\text { Avaliar o efeito } \\
\text { dos fatores } \\
\text { de risco } \\
\text { cardiovascular } \\
\text { na deficiência } \\
\text { auditiva. }\end{array}$ & $\begin{array}{l}\text { Estudo transversal, do qual par- } \\
\text { ticiparam } 220 \text { sujeitos com idade } \\
\text { média de } 45,24 \text { ( } \pm 12,21 \text { ) anos, } \\
\text { realizado no período de dezem- } \\
\text { bro de } 2015 \text { e abril de } 2016 \text {. Um } \\
\text { questionário pré-testado foi usado } \\
\text { para obter informações sobre os } \\
\text { dados biográficos e médicos dos } \\
\text { participantes, após o qual, eles } \\
\text { foram examinados e tiveram seus } \\
\text { limiares auditivos medidos. A DM } \\
\text { e a HAS foram considerados fato- } \\
\text { res de risco cardiovascular, sendo } \\
\text { o diagnóstico destas patologias } \\
\text { realizado por um médico. } \\
\text { Foi determinada a média dos limia- } \\
\text { res audiométricos de } 500,1000 \text {, } \\
2000 \text { e } 4000 \text { Hz e categorizada de } \\
\text { acordo com os graus de compro- } \\
\text { metimento auditivo propostos pela } \\
\text { Organização Mundial da Saúde da } \\
\text { seguinte forma: audição normal } \\
\text { (<25 dB), perda auditiva leve ( } 26 \\
\text { e } 40 \text { dB), perda auditiva moderada } \\
\text { (41 e } 60 \text { dB), perda auditiva grave } \\
\text { (61 e } 80 \text { dB), profunda perda au- } \\
\text { ditiva ( } 81 \text { dB ou superior). }\end{array}$ & $\begin{array}{l}\text { A média dos limiares auditi- } \\
\text { vos para tom puro foi } 19,15 \\
( \pm 9,28) \text { dBNA. Trinta e seis } \\
(16,4 \%) \text { participantes apre- } \\
\text { sentaram algum grau de perda } \\
\text { auditiva. Além disso, } 30 \text { deles } \\
\text { ( } 13,6 \%) \text { apresentavam HAS, } \\
\text { enquanto } 23 \text { ( } 10,5 \%) \text { apre- } \\
\text { sentavam DM. Além disso, } 43 \\
\text { participantes ( } 19,5 \%) \text { tinham } \\
\text { IMC } \geq 25 \mathrm{~kg} / \mathrm{m}^{2} \text {. Nove dos } 220 \\
\text { participantes ( } 4,1 \%) \text { tinham } \\
\text { histórico de tabagismo. Sendo } \\
\text { assim, a HAS, o DM. IMC } \geq 25 \\
\text { kg/m²e envelhecimento foram } \\
\text { significativamente associados } \\
\text { ao risco de perda auditiva, } \\
\text { enquanto tabagismo e gênero } \\
\text { não. }\end{array}$ \\
\hline
\end{tabular}




\begin{tabular}{|c|c|c|}
\hline Autor/Ano & Título & Objetivo \\
\hline $\begin{array}{c}\text { Laurie Penha } \\
\text { Rolim; } \\
\text { Camila Maia } \\
\text { Rabelo; } \\
\text { Ivone } \\
\text { Ferreira } \\
\text { Neves Lobo; } \\
\text { Renata } \\
\text { Rodrigues } \\
\text { Moreira; } \\
\text { Alessandra } \\
\text { Giannella } \\
\text { Samelli / } \\
2015\end{array}$ & $\begin{array}{c}\text { Interação } \\
\text { entre diabetes } \\
\text { mellitus e } \\
\text { hipertensão } \\
\text { na audição de } \\
\text { idosos }^{11}\end{array}$ & $\begin{array}{c}\text { Comparar } \\
\text { os limiares } \\
\text { auditivos de } \\
\text { idosos com DM, } \\
\text { HAS e DM + } \\
\text { HAS com grupo } \\
\text { controle (GC). }\end{array}$ \\
\hline
\end{tabular}

Estudo retrospectivo realizado Não houve diferença estatistiatravés do levantamento de da- camente significante entre as dos de prontuários de 80 idosos orelhas, sendo estes resultacom avaliação auditiva completa dos agrupados. Comparações (anamnese, audiometria tonal, au- entre as médias dos limiares diometria vocal e imitanciometria), auditivos do GC e do DM ou HA entre os anos de 2008 e 2012. Os não apresentaram diferenças indivíduos foram divididos em qua- estatisticamente significantro grupos, com 20 sujeitos cada: tes. Entretanto, foi observada $D M, H A, D M+H A$ e sem doenças diferença estatisticamente crônicas conhecidas (GC). significante na comparação entre esses três grupos e o grupo DM + HA para várias das frequências avaliadas, sugerindo um efeito sinérgico das duas doenças crônicas sobre a audição.

\begin{tabular}{ccc}
\hline Chen-Yu & Metabolic & Examinar se \\
Chien; Shu- & Syndrome & a síndrome \\
Yu Tai; Ling- & Increases the & metabólica \\
Feng Wang; & Risk of Sudden & aumenta \\
Edward Hsi; & Sensorineural & o risco de \\
Ning-Chia & Hearing Loss & perda auditiva \\
Chang; Ming- & in Taiwan: A & neurossensorial \\
Tsang Wu; e & Case-Control & súbita em \\
Kuen-Yao Ho & Study & Taiwan.
\end{tabular}

Foram investigados retrospec- Indivíduos com síndrome metivamente, 181 casos de perda tabólica tiveram um risco auditiva neurossensorial súbita 3,54 vezes maior (intervalo e 181 casos controles de 2010 a de confiança de $95 \%$ [IC] = 2012 , comparando suas variáveis $2,00-6,43, \mathrm{P}<0,01)$ de ter clínicas. Foi analisada a relação perda auditiva neurossensoentre síndrome metabólica e perda rial súbita em comparação auditiva neurossensorial súbita. A com aqueles sem síndrome síndrome metabólica foi definida de metabólica. Com o aumento acordo com o Painel de Tratamento do número de componentes de Adultos do National Cholesterol da síndrome metabólica, o Education Programme III. risco de perda auditiva neuA avaliação audiológica incluiu rossensorial súbita aumentou. audiometria tonal liminar inicial e $A$ vertigem foi associada a um de acompanhamento, audiometria resultado ruim $(P=0,02$; IC vocal, imitanciometria, potencial $95 \%=1,13$ a 5,13, razão de evocado auditivo de tronco ence- chances ajustada $=2,39$ ). fálico e ressonância magnética. 0 padrão de perda auditiva Os audiogramas foram categori- pode influenciar o resultado da zados em quatro padrões: perda perda auditiva neurossensorial auditiva de tom baixo, tom alto, súbita $(P<0,01)$ achatado e total. A severidade da perda auditiva foi avaliada com o limiar auditivo médio em 5 frequências $(250,500,1000,2000$ e $4000 \mathrm{~Hz}$ ) no audiograma de tom puro realizado quando a perda auditiva neurossensorial súbita foi diagnosticada. A severidade foi então categorizada em 1 de 4 graus, com base no audiograma inicial, que incluía leve (perda auditiva inferior a $40 \mathrm{~dB}$ ), moderado (perda auditiva superior a $40 \mathrm{~dB}$, mas não superior a $70 \mathrm{~dB}$ ), grave (perda auditiva de superior a 70 $\mathrm{dB}$, mas não superior a $90 \mathrm{~dB}$ ) e profunda (perda auditiva superior a $90 \mathrm{~dB}$ ). A melhora da audição foi avaliada comparando o último audiograma de acompanhamento do paciente com o audiograma realizado quando perda auditiva súbita foi diagnosticada. Todos os pacientes foram acompanhados por pelo menos 3 meses. 


\begin{tabular}{ccccc}
\hline Autor/Ano & Título & Objetivo & Metodologia & Resultados \\
\hline Abdulbari & Interaction & Determinar a & Durante o período do estudo A maioria dos casos de perda \\
Bener; & between & prevalência de & (janeiro 2013 a julho de 2014), auditiva foi observada acima \\
Abdulla OAA & diabetes & perda auditiva e a prevalência, audição, teste au- dos 45 anos $(44,8 \%$ vs $51,7 \%$, & (1) \\
Al-Hamaq; & mellitus and & sua associação & diológico, história familiar e pro- $p=0,05)$. Os casamentos \\
Khalid & hypertension & com DM2 em & blemas médicos associados à consanguíneos foram mais \\
Abdulhadi; & on risk of & uma população & deficiência auditiva em pacientes observados na perda auditiva
\end{tabular}

Ahmed $\mathrm{H}$. hearing loss

Salahaldin; in highly Loida endogamous

Gansan/2017 population ${ }^{\text {13 }}$ altamente endogâmica. de meia-idade foram registrados. $(32,1 \%)$ do que na audição A audição foi avaliada por meio normal $(21,8 \%)(p=0,028)$. da audiometria tonal e conside- A média da duração do início rada normal quando os limiares do diabetes ( $7,8 \pm 4,12$ anos), encontravam-se até $25 \mathrm{~dB}$, sendo distúrbio do sono $(5,81 \pm 1,29$ as perda auditivas classificadas em $\mathrm{h}$ ), indivíduos obesos $(38 \%$ leve (26 a $40 \mathrm{~dB}$ ), moderada (41 a vs 27,4\%); história familiar $55 \mathrm{~dB})$, grave (56 a $70 \mathrm{~dB})$, muito de diabetes $(30,6$ vs $23,1 \%)$ grave (71 a $90 \mathrm{~dB}$ ), profunda (91- foi maior entre os deficientes $120 \mathrm{~dB}$ ) e surdo (sem audição). auditivos. Os fatores de risco associados foram significativamente maiores no DM2 com PA, do que em DM com audição normal. Houve diferença entre a deficiência auditiva e a audição normal no DM2 quanto à frequência de audição $(p<0,001)$. Adultos com DM e hipertensão associados apresentavam maior comprometimento auditivo em populações altamente endogâmico.

\begin{tabular}{|c|c|c|c|c|}
\hline $\begin{array}{l}\text { Caroline Luiz } \\
\text { Meneses- } \\
\text { Barriviera ; } \\
\text { Jéssica } \\
\text { Aparecida } \\
\text { Bazoni ; } \\
\text { Marcelo Yugi } \\
\text { Doi ; } \\
\text { Luciana } \\
\text { Lozza de } \\
\text { Moraes } \\
\text { Marchiori / } \\
2018\end{array}$ & $\begin{array}{c}\text { Probable } \\
\text { Association of } \\
\text { Hearing Loss, } \\
\text { Hypertension } \\
\text { and Diabetes } \\
\text { Mellitus in the } \\
\text { Elderly }^{14}\end{array}$ & $\begin{array}{c}\text { Avaliar a } \\
\text { prevalência de } \\
\text { perda auditiva } \\
\text { e sua possível } \\
\text { associação com } \\
\text { HAS e DM em } \\
\text { idosos. }\end{array}$ & $\begin{array}{l}\text { Estudo transversal com } 519 \text { idosos } \\
\text { com idade superior a } 60 \text { anos, sub- } \\
\text { metidos à avaliação audiológica, e } \\
\text { responderam a um questionário } \\
\text { de comorbidades. A variável de- } \\
\text { pendente foi a presença de perda } \\
\text { auditiva. As variáveis indepen- } \\
\text { dentes foram idade, gênero, DM } \\
\text { e hipertensão. As variáveis foram } \\
\text { apresentadas em números abso- } \\
\text { lutos e proporções e permitiram } \\
\text { estimar a prevalência. } \\
\text { A avaliação audiológica foi com- } \\
\text { posta por anamnese, otoscopia e } \\
\text { audiometria tonal. Para classificar } \\
\text { as perdas auditivas foi utilizada a } \\
\text { classificação de Davis e Silverman } \\
\text { (1970) para analisar a média au- } \\
\text { ditiva I ( } 500 \mathrm{~Hz}, 1000 \text { Hz e } 2000 \\
\text { Hz) e a classificação do National } \\
\text { Committee on Noise e do estudo } \\
\text { de Amorim et al. (2008) para ana- } \\
\text { lisar a média auditiva II (3000 Hz, } \\
4000 \mathrm{~Hz} \text { e } 6000 \mathrm{~Hz}) \text {. Os graus de } \\
\text { perda auditiva foram classificados } \\
\text { em: leve (26 a } 40 \mathrm{~dB} \text { ), moderado } \\
\text { (41 a } 70 \mathrm{~dB} \text { ), grave ( } 71 \text { a } 90 \mathrm{~dB} \text { ) } \\
\text { e profundo ( } 91 \mathrm{~dB} \text { ). }\end{array}$ & $\begin{array}{l}\text { A perda auditiva neurossen- } \\
\text { sorial foi mais prevalente } \\
(66,26 \%) \text {, sendo frequente- } \\
\text { mente bilateral }(91,56 \%) \text { e } \\
\text { de grau leve }(26,50 \%) \text {. A DM } \\
\text { associou-se à perdas auditivas } \\
\text { em altas frequências em } \\
\text { idosos e, segundo a regressão } \\
\text { logística múltipla, os fatores } \\
\text { de risco são independentes } \\
\text { da perda auditiva apenas para } \\
\text { idade e exposição ao ruído } \\
\text { ocupacional. }\end{array}$ \\
\hline
\end{tabular}




\begin{tabular}{|c|c|c|c|c|}
\hline Autor/Ano & Título & Objetivo & Metodologia & Resultados \\
\hline $\begin{array}{l}\text { Em-Hwan } \\
\text { Oh; Jong } \\
\text { Hoon Lee; } \\
\text { Park Dong } \\
\text { Choon; } \\
\text { MyungGu } \\
\text { Kim; Ji } \\
\text { Hyung } \\
\text { Chung; Sang } \\
\text { Hoon Kim; } \\
\text { Seung Geun } \\
\text { Yeo / } 2014\end{array}$ & $\begin{array}{c}\text { Hearing Loss } \\
\text { as a Function } \\
\text { of Aging and } \\
\text { Diabetes } \\
\text { Mellitus: A } \\
\text { Cross Sectional } \\
\text { Study }{ }^{15}\end{array}$ & $\begin{array}{c}\text { Avaliar as } \\
\text { contribuições do } \\
\text { DM e da HAS, } \\
\text { doenças crônicas } \\
\text { associadas ao } \\
\text { envelhecimento, } \\
\text { bem como } \\
\text { o próprio } \\
\text { envelhecimento, } \\
\text { para a perda } \\
\text { auditiva em } \\
\text { exames de } \\
\text { triagem de } \\
\text { saúde. }\end{array}$ & $\begin{array}{l}\text { Este estudo incluiu } 37.773 \text { indi- } \\
\text { víduos submetidos a exames de } \\
\text { triagem de saúde de } 2009 \text { a } 2012 \text {. } \\
\text { Foram avaliadas as relações entre } \\
\text { limiar auditivo e idade, limiar audi- } \\
\text { tivo a cada frequência com base na } \\
\text { faixa etária, grau da perda auditiva } \\
\text { e presença ou ausência de HAS e } \\
\text { DM. Os limiares auditivos foram } \\
\text { avaliados nas frequências de } 500 \text {, } \\
1000,2000,3000,4000 \text { e } 6000 \mathrm{~Hz} \text {. } \\
\text { Um limiar auditivo médio } \geq 26 \mathrm{~dB} \\
\text { foi definido como perda auditiva } \\
\text { e o grau de perda auditiva foi } \\
\text { classificado em leve ( } 26,40 \mathrm{~dB} \text { ), } \\
\text { moderado ( } 41,55 \mathrm{~dB}) \text {, moderada- } \\
\text { mente grave ( } 56,70 \mathrm{~dB} \text { ) e grave } \\
\text { ( } 71,90 \text { dB) com base no padrão } \\
\text { ISO. O limiar auditivo médio em } \\
\text { cada faixa etária e o limiar auditivo } \\
\text { em cada frequência também foram } \\
\text { determinados. }\end{array}$ & $\begin{array}{l}\text { A prevalência de perda auditiva } \\
\text { aumentou com a idade, sendo } \\
1,6 \%, 1,8 \%, 4,6 \%, 14,0 \% \text {, } \\
30,8 \% \text { e } 49,2 \% \text { nos indivíduos } \\
\text { de } 20,30,40,60, \text { e } 70 \text { anos, } \\
\text { respectivamente }(\mathrm{p}<0,05) .0 \\
\text { valor da audição por frequên- } \\
\text { cia mostrou alterações base- } \\
\text { adas no envelhecimento, na } \\
\text { ordem de } 6000,4000,2000 \text {, } \\
1000 \text { e } 500 \mathrm{~Hz} \text {, indicando } \\
\text { maiores perdas auditivas nas } \\
\text { altas frequências. O grau de } \\
\text { perda auditiva variou de leve } \\
\text { a grave. O envelhecimento } \\
\text { e DM foram correlacionados } \\
\text { com a prevalência de perda } \\
\text { auditiva ( } p<0,05) \text {. Não houve } \\
\text { associação estatisticamente } \\
\text { significante entre perda audi- } \\
\text { tiva e hipertensão após ajuste } \\
\text { por idade e DM. }\end{array}$ \\
\hline $\begin{array}{l}\text { Stephen } \\
\text { Oluwatosin } \\
\text { Adebola; } \\
\text { Micheal A. } \\
\text { Olamoyegun; } \\
\text { Olusola A. } \\
\text { Sogebi; } \\
\text { Sandra O. } \\
\text { Iwuala; } \\
\text { John A. } \\
\text { Babarinde; } \\
\text { Abayomi O. } \\
\text { Oyelakin / } \\
2016\end{array}$ & $\begin{array}{l}\text { Otologic and } \\
\text { audiologic } \\
\text { characteristics } \\
\text { of type } 2 \\
\text { diabetics in a } \\
\text { tertiary health } \\
\text { institution in } \\
\text { Nigeria }^{16}\end{array}$ & $\begin{array}{l}\text { Descrever } \\
\text { o padrão } \\
\text { de doenças } \\
\text { otológicas } \\
\text { e acuidade } \\
\text { auditiva em } \\
\text { pacientes } \\
\text { com DM tipo } \\
2 \text { (DM2), } \\
\text { comparando } \\
\text { esses padrão } \\
\text { com o de não } \\
\text { diabéticos, } \\
\text { e também } \\
\text { explorar } \\
\text { os seus } \\
\text { determinantes. }\end{array}$ & $\begin{array}{l}\text { Noventa e sete pacientes com o } \\
\text { diagnóstico clínico de diabetes } \\
\text { mellitus (DM2), foram pareados } \\
\text { por sexo e idade com } 90 \text { pacientes } \\
\text { não diabéticos ( } 180 \text { orelhas). Es- } \\
\text { ses pacientes foram avaliados por } \\
\text { meio de otoscopia e audiometria } \\
\text { tonal liminar, durante um período } \\
\text { de } 6 \text { meses. }\end{array}$ & $\begin{array}{l}\text { O estudo relatou uma taxa de } \\
\text { prevalência de } 21,6 \% \text { de perda } \\
\text { auditiva (PA) em pacientes } \\
\text { com DM2. O tipo mais comum } \\
\text { de doença otológica que apre- } \\
\text { sentou associação significativa } \\
\text { com pacientes com DM2 foi a } \\
\text { otite média com efusão ( } p= \\
0,027) \text {. O DM2 foi significati- } \\
\text { vamente associado a achados } \\
\text { audiométricos alterados ( } p \\
=0,022), \text { principalmente PA } \\
\text { neurossensorial ( } p=0,012), \\
\text { do grau moderado }(p=0,057) \text {. } \\
\text { Hipertensão coexistente e } \\
\text { controle glicêmico deficiente } \\
\text { estiveram significativamente } \\
\text { associados ao agravamento } \\
\text { da audição dos pacientes com } \\
\text { DM2 ( } p<0,001 \text { e } p=0,009, \\
\text { respectivamente). }\end{array}$ \\
\hline $\begin{array}{c}\text { Laurie Penha } \\
\text { Rolim; } \\
\text { Alessandra } \\
\text { Giannella } \\
\text { Samelli; } \\
\text { Renata } \\
\text { Rodrigues } \\
\text { Moreira; } \\
\text { Carla Gentile } \\
\text { Matas; } \\
\text { Itamar } \\
\text { de Souza } \\
\text { Santos; } \\
\text { Isabela } \\
\text { Martins } \\
\text { Bensenor; } \\
\text { Paulo } \\
\text { Andrade } \\
\text { Lotufo / 2017 }\end{array}$ & $\begin{array}{l}\text { Effects of } \\
\text { diabetes } \\
\text { mellitus and } \\
\text { systemic } \\
\text { arterial } \\
\text { hypertension } \\
\text { on elderly } \\
\text { patients' } \\
\text { hearing }^{17}\end{array}$ & $\begin{array}{l}\text { Comparar a } \\
\text { audiometria } \\
\text { inicial (A1) } \\
\text { com uma } \\
\text { audiometria } \\
\text { subsequente } \\
\text { (A2) realizada } \\
\text { após um } \\
\text { intervalo de } 3 \\
\text { a } 4 \text { anos em } \\
\text { uma população } \\
\text { de idosos } \\
\text { com diabetes } \\
\text { mellitus e / ou } \\
\text { hipertensão } \\
\text { arterial } \\
\text { sistêmica, }\end{array}$ & $\begin{array}{l}\text { Participaram do estudo } 100 \text { ido- } \\
\text { sos com diagnóstico de DM e/ou } \\
\text { HAS, divididos em quatro grupos: } \\
\text { Grupo DM ( } n=20) \text {; Grupos HAS } \\
\text { ( } n=20) \text {; Grupo DM/HAS ( } n=20) \text {; } \\
\text { Grupo controle ( } n=40) \text {. Todos os } \\
\text { participantes deveriam ter uma } \\
\text { avaliação audiológica completa } \\
\text { prévia (A1). Uma nova avaliação } \\
\text { audiológica (A2) foi realizada } 3-4 \\
\text { anos após a primeira. As avaliações } \\
\text { audiológicas foram compostas de } \\
\text { anamnese, meatoscopia, imitan- } \\
\text { ciometria, audiometria tonal e } \\
\text { audiometria vocal. Os participantes } \\
\text { com DM e/ou HAS foram pareados } \\
\text { na proporção } 1: 1 \text { com participan- } \\
\text { tes de mesmo sexo e idade, sem } \\
\text { essas condições clínicas. Para a } \\
\text { comparação dos limiares auditivos } \\
\text { de A1 com A2 entre os grupos es- } \\
\text { tudo e seu respectivo controle, foi } \\
\text { considerado o aumento médio dos } \\
\text { limiares auditivos por ano. }\end{array}$ & $\begin{array}{l}\text { Ao comparar o aumento médio } \\
\text { anual nos limiares auditivos da } \\
\text { A1 com a A2, considerando } \\
\text { cada grupo de estudo e seu } \\
\text { respectivo controle, pode- } \\
\text {-se observar que não houve } \\
\text { diferença estatisticamente } \\
\text { significante para nenhuma } \\
\text { das frequências para o grupo } \\
\text { DM; para o grupo com HAS, } \\
\text { diferenças significativas fo- } \\
\text { ram observadas após } 4 \mathrm{kHz} \text {. } \\
\text { Para o grupo com DM e HAS, } \\
\text { diferenças significativas foram } \\
\text { observadas nas frequências } \\
\text { de } 500,2 \mathrm{kHz}, 3 \mathrm{kHz} \text { e } 8 \mathrm{kHz} \text {. }\end{array}$ \\
\hline
\end{tabular}




\begin{tabular}{|c|c|c|c|c|}
\hline Autor/Ano & Título & Objetivo & Metodologia & Resultados \\
\hline $\begin{array}{l}\text { Alessandra } \\
\text { G. Samelli; } \\
\text { Itamar S. } \\
\text { Santos; } \\
\text { Renata R. } \\
\text { Moreira; } \\
\text { Camila M. } \\
\text { Rabelo; } \\
\text { Laurie P. } \\
\text { Rolim; } \\
\text { Isabela J. } \\
\text { Bensenõr; } \\
\text { Paulo A. } \\
\text { Lotufo / } 2017\end{array}$ & $\begin{array}{c}\text { Diabetes } \\
\text { mellitus and } \\
\text { sensorineural } \\
\text { hearing loss: } \\
\text { is there an } \\
\text { association? } \\
\text { Baseline of the } \\
\text { Brazilian } \\
\text { Longitudinal } \\
\text { Study of Adult } \\
\text { Health (ELSA- } \\
\text { Brasil) }{ }^{18}\end{array}$ & $\begin{array}{c}\text { Investigar a } \\
\text { associação } \\
\text { entre diabetes } \\
\text { e perda auditiva } \\
\text { neurossensorial. } \\
\text { Nós avaliamos } \\
\text { a influência } \\
\text { do tempo do } \\
\text { diagnóstico de } \\
\text { diabetes nesta } \\
\text { associação após } \\
\text { o controle para } \\
\text { diagnóstico de } \\
\text { idade, gênero } \\
\text { e hipertensão } \\
\text { e excluímos os } \\
\text { indivíduos com } \\
\text { exposição ao } \\
\text { ruído. }\end{array}$ & $\begin{array}{l}\text { Este estudo transversal avaliou } 901 \\
\text { adultos e idosos participantes do } \\
\text { Estudo Longitudinal de Saúde do } \\
\text { Adulto (ELSA-Brasil). A avaliação } \\
\text { audiológica foi composta por audio- } \\
\text { metria tonal liminar, audiometria } \\
\text { vocal e imitanciometria. Não foram } \\
\text { encontradas diferenças significati- } \\
\text { vas entre as orelhas esquerda e } \\
\text { direita, sendo elas agrupadas. Fo- } \\
\text { ram calculados os valores médios } \\
\text { para cada frequência audiométrica } \\
\text { (limiar por frequência); frequên- } \\
\text { cias de faixa baixa a média ( } 250 \\
\text { a } 2000 \text { Hz); frequências de alto } \\
\text { alcance (3000-8000 Hz); limiar de } \\
\text { reconhecimento de fala e índice de } \\
\text { reconhecimento de fala. O padrão } \\
\text { de normalidade considerado para } \\
\text { os limiares por frequência foi } 25 \\
\text { dBNA. }\end{array}$ & $\begin{array}{l}\text { Os limiares auditivos e os } \\
\text { resultados dos testes de fala } \\
\text { foram significativamente pio- } \\
\text { res no grupo com diabetes do } \\
\text { que no grupo sem diabetes. } \\
\text { No entanto, não foram encon- } \\
\text { tradas diferenças significativas } \\
\text { entre os participantes com e } \\
\text { sem diabetes após o ajuste } \\
\text { para idade, gênero e presença } \\
\text { de hipertensão. Os limiares } \\
\text { auditivos não foram afetados } \\
\text { pela exposição ocupacional ao } \\
\text { ruído nos grupos com e sem } \\
\text { diabetes. Além disso, nenhuma } \\
\text { associação entre a duração } \\
\text { do diabetes e os limiares } \\
\text { auditivos foi observada após } \\
\text { o ajuste para idade, gênero e } \\
\text { hipertensão. }\end{array}$ \\
\hline
\end{tabular}

Legenda: DM = Diabetes Mellitus; HAS = Hipertensão Arterial Sistêmica; IMC = índice de massa corporal; GC = Grupo Controle; DM2 $=$ Diabetes Mellitus tipo 2 .

\section{Discussão}

Nesta pesquisa verificou-se que a DM e a HAS são consideradas fatores de risco para a perda auditiva $^{10-17}$. Além das DCNTs pesquisadas, outros fatores de risco foram citados como relacionados ao surgimento da perda auditiva como o tabagismo e o elevado $\mathrm{IMC}^{7,10,12}$.

Apesar de ter-se constatado grande variabilidade com relação à idade dos sujeitos das pesquisas selecionadas ${ }^{10,13,15,16,18}$ foi possível observar o predomínio de idosos, visto que praticamente todos os estudos tinham indivíduos com idade igual ou superar a 60 anos em suas amostras ${ }^{10-12,14-18}$. Isto pode ser justificado pelo fato da HAS ser uma doença altamente prevalente entre as pessoas idosas, acometendo cerca de 50 a $70 \%$ das pessoas nessa faixa etária, bem como a DM, que é uma das doenças crônicas mais comuns nessa faixa de idade ${ }^{19}$. Além disso, o envelhecimento está diretamente associado com o aumento dos limiares auditivos, resultando em um maior grau de perda auditiva em indivíduos mais velhos ${ }^{14,15,20-22}$. Dessa forma, a idade pesquisada é um fator extremamente relevante na análise dos resultados.

Estudo publicado recentemente corrobora com os achados supracitados, visto que os idosos que apresentaram DM e HAS como principais comorbidades queixaram-se mais frequentemente de hipoacusia $(40,3 \%)$ e zumbido $(36,6 \%)$, sendo a perda auditiva sensorioneural a mais comum nessa população ${ }^{22}$. Para mensurar o grau/nível de DM, da HAS e a presença ou não de perda auditiva, alguns procedimentos foram realizados, como a audiometria tonal liminar ${ }^{10-18}$, logoaudiometria e imitanciomatria ${ }^{11,12,17,18}$, exame de sangue ${ }^{13,15,16,18}$ para confirmação da DM e, por fim, aferição da pressão arterial ${ }^{10-18}$ procedimentos que corroboram em parte com os realizados em outros estudos compilados na literatura ${ }^{8,20,23}$.

Em relação à avaliação audiológica, existe consenso na literatura quanto à forma ideal de se realizar a audiometria tonal liminar, sendo as recomendações seguidas na maioria dos estudos selecionados ${ }^{11,13,14,16-18}$, bem como evidenciado em outras pesquisas compulsadas na literatura ${ }^{20,23}$. Em contrapartida, registrou-se um estudo cujos autores optaram por avaliar as frequências de 500 a 6000 $\mathrm{kHz}^{15} \mathrm{e}$ outros dois que não deixaram claro a faixa de frequências avaliadas, apesar de um deles referir que a média para classificação da perda auditiva foi realizada com base nos limiares auditivos de 500 a $4000 \mathrm{~Hz}^{10} \mathrm{e}$ o outro mencionar que cinco frequências $(250,500,1000,2000 \mathrm{e} 4000 \mathrm{~Hz})$ foram usadas na avaliação da gravidade da perda auditiva ${ }^{12}$. Já a realização de logoaudiometria e imitanciometria não foi consenso, bem como verificado dentre as publicações, cujos exames são realizados em menor proporção. 
Dois procedimentos que não foram realizados em nenhum dos estudos selecionados e são de extrema importância, levando em consideração os achados obtidos por alguns estudos, que constataram perda auditiva principalmente nas altas frequências ${ }^{14,15,17}$, são a audiometria de altas frequências e as emissões otoacústicas. Tais exames auxiliam no diagnóstico precoce, ou seja, antes do comprometimento atingir as frequências utilizadas na detecção e compreensão da fala. Além da DM e HAS, outros fatores de risco foram mencionados, como exposição ao ruído ${ }^{14,18}$, histórico familiar de diabetes e predisposição para desenvolver outras doenças ${ }^{13}$. Nesta vertente, vale ressaltar a importância da prevenção desses fatores de risco por meio de ações de prevenção em saúde, diminuindo o risco do aparecimento da perda auditiva $a^{2,7,9,10}$.

Em relação ao tempo em que os pacientes já possuem o diagnóstico de HAS e/ou DM, este é em média de cinco a 16 anos para ambas comorbidades ${ }^{16,17}$. Quanto à idade em que os sujeitos receberam o diagnóstico, esta variou de 30 a 72 anos de idade em um dos estudos com enfoque no $\mathrm{DM}^{18}$, enquanto em outro, os portadores de DM receberam o diagnóstico com 61,75 anos de idade em média e o diagnóstico de HAS foi dado com 52,65 anos de idade, em média ${ }^{17}$, não sendo observada relação entre a idade e o tempo de diagnóstico de HAS e/ ou DM com a perda auditiva. No entanto, é importante ressaltar que tanto o DM quanto a HAS são doenças que podem ser assintomáticas e, portanto, o tempo de início da doença pode ser maior do que o referido pelos indivíduos.

Os indivíduos hipertensos apresentaram probabilidades significativamente aumentadas de desenvolver uma perda auditiva, quando comparado aos indivíduos não hipertensos ${ }^{10}$, isso possivelmente em virtude da hipertensão causar insuficiência microcirculatória que pode determinar uma maior ou menor deterioração do sistema auditivo periférico, podendo causar o aparecimento de perdas auditivas súbitas ou progressivas.

Comparando o grupo de portadores de DM ao grupo controle, em três diferentes estudos pode-se verificar que os participantes com DM apresentaram os limiares auditivos elevados ${ }^{11,16,17}$. Ratificando tais achados, estudo similar verificou piora nos limiares auditivos dos sujeitos com DM, sendo esses achados significantes quando comparados ao grupo controle, indicando a correlação entre $\mathrm{DM}_{\text {e perda auditiva }}{ }^{24}$. Dessa forma, estima-se que portadores de DM possuem pré-disposição ao surgimento da perda auditiva ${ }^{22}$. Acredita-se que uma das implicações da DM seja a angiopatia, que pode interferir no suprimento de nutrientes e oxigênio da cóclea. Além das alterações cocleares, o DM também pode causar degeneração secundária do oitavo nervo craniano (VII), provocando perdas auditivas neurais ${ }^{1,5}$.

Dois estudos ${ }^{11,17}$ compararam um grupo com HAS e DM associados com um grupo controle e observaram que o grupo com HAS e DM foi o que apresentou os limiares auditivos mais alterados, indo ao encontro do constatado em estudo desenvolvido em uma população altamente endogâmica que concluiu que adultos com DM e HAS associados apresentaram maior comprometimento auditi$\mathrm{vo}^{13}$. Dessa forma, pode-se dizer que indivíduos que possuem HAS e DM estão mais suscetíveis a desenvolver uma perda auditiva. Em outro estudo ${ }^{16}$ a deficiência auditiva em pacientes com DM2 foi agravada pela hipertensão coexistente e pelo controle glicêmico inadequado.

Conforme análise dos estudos, os indivíduos apresentaram mais alterações nas frequências altas $^{14,15,17}$, sendo a perda auditiva bilateral ${ }^{14}$, de grau leve ${ }^{14}$ ou moderado ${ }^{16}$. A associação do DM e da HAS com o grau da perda auditiva é um aspecto pouco abordado nas publicações atuais, sendo necessário o desenvolvimento de estudos que avaliem uma gama maior de frequências e determinem o grau da alteração, tanto para baixas e médias quanto para altas frequências.

Desta forma, não há consenso e detalhamento sobre os reais fatores que contribuem para o risco do surgimento da perda auditiva em sujeitos com DM e HAS, sendo necessária a realização de novas pesquisas. Sugere-se o desenvolvimento de estudos que avaliem altas frequências $(>8000 \mathrm{~Hz})$ e estudem as emissões otoacústicas (EOA), bem como o potencial evocado auditivo de tronco encefálico (PEATE) desses pacientes, já que acredita-se que a DM e a HAS podem provocar alterações cocleares, bem como centrais.

\section{Conclusão}

A partir dos estudos analisados pode-se concluir que existe relação entre DM e HAS com a perda auditiva, tanto em adultos quanto em idosos, que representam a parcela que mais cresce na população brasileira atualmente. $\mathrm{O}$ comprometi- 
mento auditivo mostrou-se maior nos sujeitos que apresentavam DM e HAS associados, sugerindo um efeito sinérgico das duas doenças crônicas sobre a audição. A alteração auditiva do tipo neurossensorial prevaleceu.

Além disso, diante dos resultados apresentados nesses indivíduos que possuem tais fatores de risco, é necessário efetivação de políticas públicas e medidas preventivas, a fim de minimizar a prevalência e as implicações da deficiência auditiva na saúde dessa população, bem como a ampliação do acesso aos serviços de saúde disponíveis à população, para que estes recebam o atendimento adequado.

Apesar de existirem muitos estudos a respeito desse assunto, ainda não foi possível estabelecer a existência de uma relação causal entre DM e/ou HAS e a perda auditiva. Sugere-se o desenvolvimento de novos estudos a fim de estabelecer essa relação, sendo estes desenvolvidos com a avaliação de uma gama maior de frequências, bem como com utilização de procedimentos como EOA e PEATE.

\section{Referências}

1. Soares MO, Goulart BNG. Associação entre deficiência auditiva, estilo de vida e doenças crônicas não transmissíveis autorreferidas no Brasil: dados da Pesquisa Nacional de Saúde [dissertação de mestrado]. LUME Repositório Digital.Porto Alegre: Universidade Federal do Rio Grande do Sul; 2016.

2. Malta DC, Bernal RTI, Lima MG, Araújo SSC, Silva MMA, Freitas MIF et al. Doenças não transmissíveis e o uso de serviços de saúde: análise da Pesquisa Nacional de Saúde no Brasil. Rev Saúde Pública. 2017; 51(1): 327-45.

3. Malta DC, Stopa SR, Szwarcwald CL, Gomes NL, Júnior JBS, Reis AAC. A vigilância e o monitoramento das principais doenças crônicas não transmissíveis no Brasil - Pesquisa Nacional de Saúde. Rev Bras Epidemiol. 2015; 18(2): 3-16.

4. Brasil. Ministério da Saúde. Caderno de Atenção Básica: Diabetes Mellitus. 2006. $\mathrm{n}^{\circ} 16$.

5. Ferreira JM, Câmara MFS, Almeida PC, Neto JB, Silva CAB. Características audiológicas de pacientes com diabetes mellitus Tipo 2. CEFAC. 2016; 18(5): 1050-9.

6. Costa MFFL, Peixoto SV, César CC, Malta DC, Moura EC. Comportamentos em saúde entre idosos hipertensos, Brasil, 2006. Rev Saúde Pública. 2009; 43(2): 18-26.

7. OMS. Organização Mundial da Saúde. Doenças crônicas não transmissíveis causam 16 milhões de mortes prematuras todos os anos. 2015.

8. Marchiori LLM, Filho EAR, Matsuo T. Hipertensão como fator associado à perda auditiva. Rev Bras Otorrinolaringol. 2006; 72(4): 533-40.

9. Moher, D. et al. Preferred reporting items for systematic review and meta-analysis protocols (PRISMA-P) 2015 statement. Syst Rev. 2015; 4:1.
10. Yikawe SS, Iseh KR, Sabir AA, Inoh MI, Solomon JH, Aliyu N. Cardiovascular risk factors and hearing loss among adults in a tertiary center of Northwestern Nigeria. World J Otorhinolaryngol Head Neck Surg. 2018; 253-257.

11. Rolim LP, Rabelo CM, Lobo IFN, Moreira RR, Samelli AG. Interação entre diabetes mellitus e hipertensão arterial sobre a audição de idosos. CoDAS. 2015; 27(5): 428-32.

12. Chien CY, Tai SY, Wang LF, Hsi E, Chang NC, Wu MT et al. Metabolic Syndrome Increases the Risk of Sudden Sensorineural Hearing Loss in Taiwan. Otolaryngology-head And Neck Surgery. 2015; 153(1): 105-11.

13. Bener A, Al-Hamaq AOAA, Abdulhadi K, Salahaldin $\mathrm{AH}$, Gansan L. Interaction between diabetes mellitus and hypertension in the risk of hearing loss in a highly endogamous population. Diabetes Metab Syndr. 2017; 8(11): 45-51.

14. Menezes-Barriviera CL, Bazoni JA, Doi MY, Marchiori LLM. Probable Association of Hearing Loss, Hypertension and Diabetes Mellitus in the Elderly. Int Arch Otorhinolaryngol. 2018; 22(4): 337-41.

15. Oh IH, Lee JH, Park DC, Kim MG, Chung JH, Kim SH et al. Hearing Loss as a Function of Aging and Diabetes Mellitus: A Cross Sectional Study. PLoS One. 2014; 9(12).

16. Adebola SO, Olamoyegun MA, Sogebi OA, Iwuala SO, Babarinde JA, Oyelakin AO. Otologic and audiologic characteristics of type 2 diabetics in a tertiary health institution in Nigeria. Braz J Otorhinolaryngol. 2016; 82(5): 567-73.

17. Rolim LP, Samelli AG, Moreira RR, Matas CG, Santos IS, Bensenor IM et al. Effects of diabetes mellitus and systemic arterial hypertension on elderly patients' hearing. Braz J Otorhinolaryngol. 2018; 84(6): 754-63.

18. Samelli AG, Santos IS, Moreira RR, Rabelo CM, Rolim LP, Bensenõr IJ et al. Diabetes mellitus and sensorineural hearing loss: is there an association? Baseline of the Brazilian Longitudinal Study of Adult Health (ELSA-Brasil). Clinics. 2017; 72(1): 5-10.

19. Brasil. Ministério da Saúde. Secretaria de Atenção à Saúde. Departamento de Atenção Básica. Envelhecimento e saúde da pessoa idosa (Cadernos de Atenção Básica, n. 19) Brasília: Ministério da Saúde, 2006.

20. Oliveira IS, Etcheverria AK, Olchik MR, Gonçalves AK, Seimetz BM, FloresLS et al. Audição em adultos e idosos: associação com sexo, idade e cognição. CEFAC. 2014; 16(5): 1463-70.

21. Pallo PAO, Levy NM, Pereira RMR, Shinjo SK. Policondrite recidivante: prevalência de doenças cardiovasculares e seus fatores de risco e características gerais da doença de acordo com o gênero. Rev Bras Reumatol Engl Ed. 2017; 57(4): 338-45.

22. Barbosa HJC, Aguiar RA, Bernardes HMC, Junior RRA, Braga DB, Szpilman ARM. Perfil clínico epidemiológico de pacientes com perda auditiva. Journal of Health and Biological Sciences. 2018; 6(4): 424-30.

23. Ferreira JM, Câmara MFS, Almeida PC, Brandão NJ, Silva CAB. Alterações auditivas associadas a complicações e comorbidades no diabetes mellitus tipo 2. Audiology Communication Research. 2013; 18(4): 250-9.

24. Diniz TH, Guida HL. Hearing loss in patients with diabetes mellitus. Braz J Otorhinolaryngol. 2009; 75(4): 573-8. 